\title{
The role of GRP78 in oxidative stress induced by tunicamycin in trabecular meshwork cells
}

\author{
Fang Chai 1,2, Hong Yan², Xiquan Zhao², Juan $\mathrm{Li}^{2}$ and Cheng Pei ${ }^{1 凶}$ \\ 'Department of Ophthalmology, First Affiliated Hospital of Xi'an Jiaotong University, Xi'an, Shaanxi 710061, China; ${ }^{2}$ Department of Ophthalmol- \\ ogy, Xi'an People's Hospital (Xi'an Fourth Hospital), Shaanxi Eye Hospital, Affiliated Guangren Hospital, School of Medicine, Xi'an Jiaotong Uni- \\ versity, Xi'an, 710004, China
}

\begin{abstract}
Objective: To clarify the regulatory effect of GRP-78 induced by tunicamycin on endoplasmic reticulum (ER) stress. Methods: Tunicamycin was used to induce ER stress in trabecular meshwork cells (HTMC and GTM3). Cell apoptosis and ROS content were detected by flow cytometry to reveal the effect of tunicamycin on trabecular meshwork cells. Results: Tunicamycin could significantly increase the ROS content and the apoptosis rate in HTMC and GTM3 $(p<0.01)$. The results showed that tunicamycin could increase the $\mathrm{Ca}^{2+}$ flow in cells. Tunicamycin can also increase expression levels of GRP78,VDAC1, ATF4, PERK, elF2a, and CHOP $(p<0.01)$. Overexpression of GRP78 protected cells from ER stress. Co-IP test showed that GRP78 directly bound to elF2. These results suggest that GRP78 may play a regulatory role by regulating eIF2. Conclusion: Tunicamycin induces oxidative stress in trabecular meshwork cells, and the increase in GRP78 expression can protect the cells during ER stress by regulating elF2.
\end{abstract}

Key words: trabecular meshwork cells; tunicamycin; GRP78; Cell apoptosis

Received: 07 April, 2021; revised: 29 November, 2021; accepted: 15 December, 2021; available on-line: 02 March, 2022

凶e-mail: peich71@mail.xitu.edu.cn

Acknowledgements of Financial Support: This work was supported by the National Natural Science Foundation of China (No. 81900856) and Xi'an Fourth Hospital Research Incubation Fund (No. 2018LH-2). Abbreviations: ER, endoplasmic reticulum; GRP78, glucose regulated protein 78; PERK, protein kinase-like ER kinase; VDAC1, voltage-dependent anionchannel 1; ATF4, activating transcription factor 4; elF2a, Eukaryotic Initiation Factor 2 alpha; CHOP, C/EBP homologous protein; Co-IP, Co-immunoprecipitation; Annexin VFITC, Annexin V-Fluorescein isothiocyanate; DCFH-DA, Dichlorodihydrofluorescein acetoacetate; ROS, Reactive oxygen species; $B C A$, Bicinchoninic Acid; TBST, Tris-buffered saline Tween; ECL, electrochemiluminescent; PCR, polymerase chain reaction; PBS, Phosphate buffer saline; DAPI, 4;,6-diamidino-2-phenylindole; NTM, normal trabecular meshwork; GTM, rabecular meshwork cells in patients with glaucoma.

\section{INTRODUCTION}

A decrease in the number of trabecular meshwork cells is one of the important pathological changes in primary open-angle glaucoma (Ashworth et al., 2018). The number of trabecular cells in primary open-angle glaucoma is reported to be significantly lower than in normal people of the same age group. However, the exact mechanism by which their numbers decline is not clear (Zhu et al., 2020). Decrease in trabecular cell numbers can be caused by a variety of mechanisms, such as wear and tear, phagocytosis, cell migration, and death of inflammatory cells. Among them, cell apoptosis may be one of the most important causes. Apoptosis receptor activation and mitochondrial damage are two classical apoptosis pathways in cells. The apoptosis pathway initiated by ER stress is a new apoptosis pathway discovered recently. ER stress is a cellular self-protection mechanism that induces the unfolded protein response to protect cells from damage (Xu et al., 2017). Although ER stress is primarily a self-protective signaling pathway, high levels of ER stress can promote cell death by activating apoptosis and inflammation (Sun et al., 2018). As an important ER molecular chaperone, GRP78 plays a key role in promoting cell protein maturation and maintaining cell function and life under normal growth state (Kaira et al., 2016; Shen et al., 2002).

GRP78 is a calcium-binding protein that is affected by harmful factors that disrupt the structure or function of the ER. When subjected to harmful stimuli, the expression of GRP78 will increase, so as to achieve the protective purpose of maintaining the ER calcium homeostasis and the stability of the internal environment (Kaira et al., 2016; Lee, 2005). GRP78 transfers unfolded or misfolded proteins to sites outside the ER to maintain the normal function of protein synthesis in the ER under stress. GRP78 reduces sensitivity of the host cells to killer T cells and prevents or delays apoptosis. When stimulated by sugar starvation, GRP78 preserves limited intracellular nutrients through glycosylation. GRP78 can reduce the lethality of cytotoxic T cells to tumor cells, promote tumor formation and drug resistance, and prevent tumor cell apoptosis. GRP78 is also involved in the synthesis and transport of secretory proteins, and participates in construction of a biological signal transduction bypass system by mediating spatial configuration changes of proteins. In summary, it has been suggested in recent years that GRP78 generation and up-regulation of GRP78 expression in cells when stimulated may be an important pro-survival mechanism which has an important protective effect on cells, thereby prolonging the survival period of cells stimulated by various harmful factors and slowing down cell apoptosis (Ibrahim et al., 2019; Bailly \& Waring, 2019; Lu et al., 2020).

In this study, to further clarify the influence of tunicamycin on the trabecular meshwork cells and its molecular mechanism, trabecular meshwork cells were treated with tunicamycin to induce ER stress and apoptosis of cells, and expression of GRP78 and its molecular regulation were studied. 


\section{MATERIALS AND METHODS}

\section{Cell apoptosis detection by flow cytometry}

$5 \times 10^{5}$ cells were taken and centrifuged at $200 \times \mathrm{g}$ for 5 min. The supernatant was discarded and the cells were gently resuspended in $200 \mu \mathrm{L}$ Annexin V-FITC binding solution. The cell suspension was incubated at $37^{\circ} \mathrm{C}$, avoiding light, for $10 \mathrm{~min}$. The cells were centrifuged at $200 \times g$ for $5 \mathrm{~min}$, then the supernatant was discarded and the cells were resuspended by adding $190 \mu \mathrm{L}$ Annexin V-FITC binding solution. $10 \mu \mathrm{L}$ of propidium iodide staining solution were added to the cell suspension, and were mixed gently and placed in an ice bath away from light (C1062L, Beyotime, Shanghai, China). Annexin V-FITC binding was analyzed at excitation wavelength of $488 \mathrm{~nm}$ and emission wavelength of $350 \mathrm{~nm}$ using flow cytometry.

\section{ROS content detection by flow cytometry}

The stock DCFH-DA (Invitrogen, UK) solution was diluted with serum-free medium at 1:1000 to a final concentration of $10 \mu \mathrm{M}$. Cells were incubated with $10 \mu \mathrm{M}$ DCHF-DA in an incubator at $37^{\circ} \mathrm{C}$ for $20 \mathrm{~min}$. The cells were washed with serum-free cell culture solution for three times to remove the DCFH-DA that did not enter the cells. ROS levels can be significantly increased after 20-30 minutes of cell stimulation. Using $488 \mathrm{~nm}$ excitation wavelength and $525 \mathrm{~nm}$ emission wavelength, the fluorescence intensity before and after stimulation was detected in real time. ROS content was assessed with the Summit software.

\section{Western blot}

The trabecular meshwork cells were mixed with a lytic solution, and lysed on ice for $30 \mathrm{~min}$. The supernatant was extracted after centrifugation for $15 \mathrm{~min}$ at $13800 \times \mathrm{g}$, diluted with loading buffer, and boiled at $100^{\circ} \mathrm{C}$ for $5 \mathrm{~min}$. The protein concentration was measured by the BCA method. Proteins were separated in $10 \%$ polyacrylamide gel and then transferred to a PVDF membrane. The membrane was blocked in 5\% BSA for $1 \mathrm{~h}$. The primary antibody of anti-VDAC1 (1:8000, Abcam), primary anti-PERK antibody $(1: 1000$, CST), anti-eIF2a (1:1000, ProteinTech), anti-CHOP (1:1000, Abcam), anti-IP3R (1:1000, ProteinTech), anti-GRP78 primary antibody (1:1000, ProteinTech) and anti- $\beta$-actin (1:1000, Beyotime) were added and incubated overnight at $4^{\circ} \mathrm{C}$ - The membrane was washed with Tris-buffered saline Tween (TBST) for 3 times, $10 \mathrm{~min}$ each. Membranes were incubated with the peroxidase-conjugated secondary antibodies (goat anti-rabbit, 1:500, Abcam) at $37^{\circ} \mathrm{C}$ for $1 \mathrm{~h}$. The membrane was washed 3 times with TBST, 10 min each, and ECL reaction solution was added. BioRad developer was used to take the picture. The bands were quantitatively analyzed by Image Lab software.

\section{qPCR detection}

The reverse transcription reaction was carried out according to the TAKARA kit, and the reaction system contained RNA $(2.2 \mu \mathrm{g})$, Oligo dT, $2 \mu \mathrm{L}$; dNTP, $4 \mu \mathrm{L}$; $5 \times$ buffer, $4 \mu \mathrm{L}$; Reverse Transcriptase, $1 \mu \mathrm{L}$; RNase inhibitor, $0.5 \mu \mathrm{L}$. The reaction conditions were: $25^{\circ} \mathrm{C}$ for $5 \mathrm{~min}, 50^{\circ} \mathrm{C}$ for $15 \mathrm{~min}, 85^{\circ} \mathrm{C}$ for $5 \mathrm{~min}$, and $4^{\circ} \mathrm{C}$ for $10 \mathrm{~min}$.

The qPCR experiment was carried out according to the qPCR kit from Beijing Tsingke Biotechnology Co.,
Ltd. The reaction system contained forward Primer, $0.4 \mu \mathrm{L}$; Reverse primer, $0.4 \mu \mathrm{L}$; SYBRGreen, $10 \mu \mathrm{L}$; $\mathrm{H}_{2} \mathrm{O}, 5.2 \mu \mathrm{L}$. The reaction conditions were $50^{\circ} \mathrm{C}$ for $2 \mathrm{~min}$ and $95^{\circ} \mathrm{C}$ for $10 \mathrm{~min} ; 95^{\circ} \mathrm{C}$ for $30 \mathrm{secs}, 60^{\circ} \mathrm{C}$ for 30 secs, 40 cycles. Primers used were as follows: ATF4-F, 5'-CCAGGTTGCCCCCTTTACGTTCTTG-3'; ATF4-R, 5'-GTTCTGCTCCATCT'TCT'TCAGCT'TC-3'; PERK-F, 5'-TCATCCAGCCT'TAGCAAACC-3', PERKR, 5'ATGCT'TTCACGGTCTTGGTC-3'; CHOP-F, 5'-CTTCTCTGGCTTGGCTGACT-3'; CHOP-R, 5'-CCCT'TGGTCT'TCCTCCTCTT-3'; $\beta$-actin-F, 5'-GACCTGACTGACTACCTCATGAAGAT-3'; $\beta$-actin-R, 5'-GTCACACT'TCATGATGGAGTTGAAGG-3’.

\section{Cell immunofluorescence detection}

After the trabecular meshwork cells were treated, the cells were fixed with $4 \%$ paraformaldehyde for 15 min, and rinsed 3 times with PBS, each time for 5 min. The cells were incubated with primary antibody in an immunofluorescence blocking solution (Biyuntian, QUICK BLOCK) for 15 min. After rewarming at $37^{\circ} \mathrm{C}$ for $30 \mathrm{~min}$, the cells were washed 3 times with PBS, each time for $5 \mathrm{~min}$. Fluorescence secondary antibodies (DyLight-594 goat anti-rabbit IgG and goat anti-human $\operatorname{IgG}$ ) were added and incubated with the cells for $1 \mathrm{~h}$ at room temperature without light. The nuclei were restained with DAPI for $15 \mathrm{~min}$, and washed 3 times with PBS, each time for $5 \mathrm{~min}$. The plates were sealed with an anti-fluorescence quenching agent, observed under fluorescence microscope and photographed.

\section{Co-IP test}

Co-IP was performed according to the protocol by Invitrogen, UK. Briefly, the cells were lysed with the RIPA lysis buffer. $100 \mu \mathrm{L}$ of $50 \%$ Protein A/G Agarose working solution was added to the sample. The mixture was shaken on the horizontal shaker at $4^{\circ} \mathrm{C}$ for $10 \mathrm{~min}$. The mixture was then centrifuged at $14000 \times g$ for $15 \mathrm{~min}$ at $4^{\circ} \mathrm{C}$ and the protein $\mathrm{A} / \mathrm{G}$ agarose was removed by transferring the supernatant into a new centrifuge tube. The concentration of total protein was determined by the BCA method. Primary antibody was added to the mixture. The mixture was then shaken slowly overnight at $4^{\circ} \mathrm{C}$. The mixture was centrifuged at $14000 \times g$ to collect the precipitate and washed 3 times with pre-chilled washing buffer. The supernatant was collected for Western blot analysis.

\section{Statistical treatment}

SPSS 20.0 software was used for statistical analysis of the experimental data, and the data were expressed as mean \pm standard deviation $(\times \pm s)$. Analysis of variance was used for comparison between groups. $p<0.05$ was considered to be significant, and $p<0.01$ was considered to be very significant.

\section{RESULTS}

\section{Tunicamycin increased the apoptosis rate and ROS content of trabecular meshwork cells}

In order to clarify influence of tunicamycin on the trabecular meshwork cells, $1 \mu \mathrm{M}$ tunicamycin was added to cultured trabecular meshwork cells (HTMC and GTM3) for $24 \mathrm{~h}$. The results showed that the cell apoptosis rate in tunicamycin treated group was significantly in- 


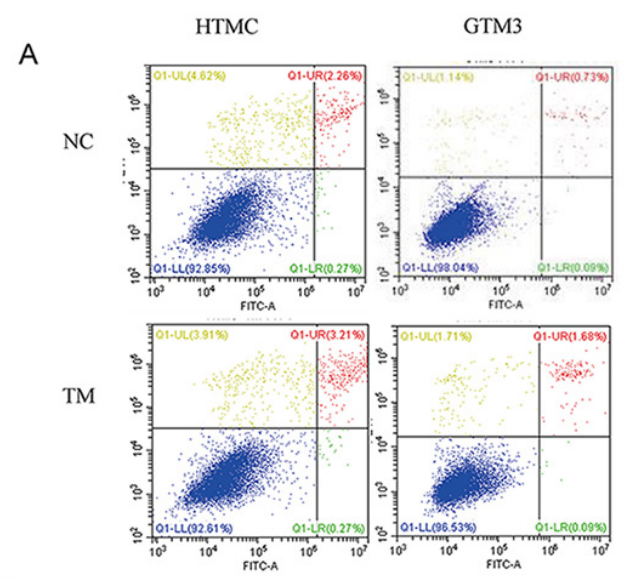

B
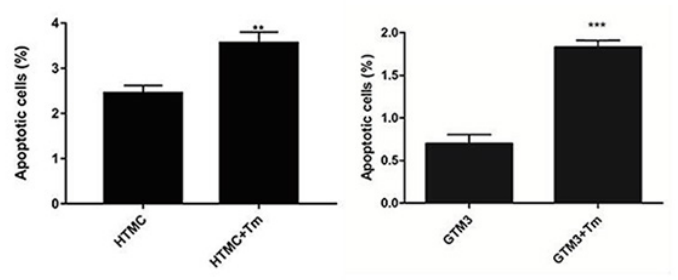

Figure 1. Tunicamycin can increase the apoptotic rate of trabecular meshwork cells.

(A) After adding tunicamycin to HTMC and GTM3 cells, the apoptosis rate was detected by flow cytometry. (B) Apoptosis rate in HTMC and GTM3 cells. ${ }^{* *} p<0.01 ;{ }^{* * *} p<0.001$. NC, negative control (PBS treated group); TM, tunicamycin treated group.

A
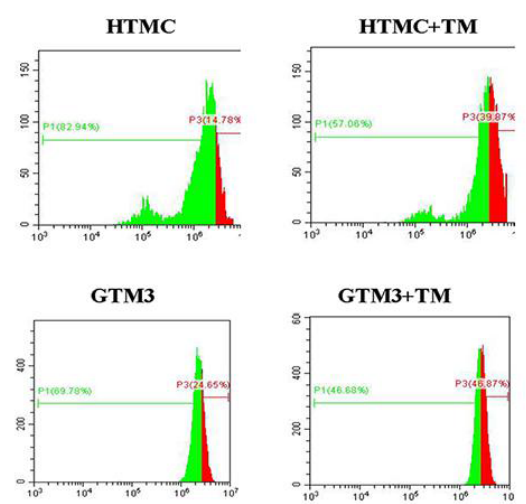

B

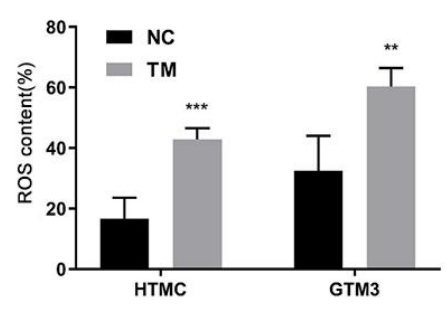

Figure 2. Tunicamycin can increase ROS content in trabecular meshwork cells.

(A) ROS content was detected by loss cell assay after tunicamycin was added to HTMC and GTM3 cells. (B) Statistical results of ROS content in HTMC and GTM3 cells. ${ }^{* *} p<0.01 ;{ }^{* * *} p<0.001$. NC, negative control (PBS treated group); TM, tunicamycin treated group.
A
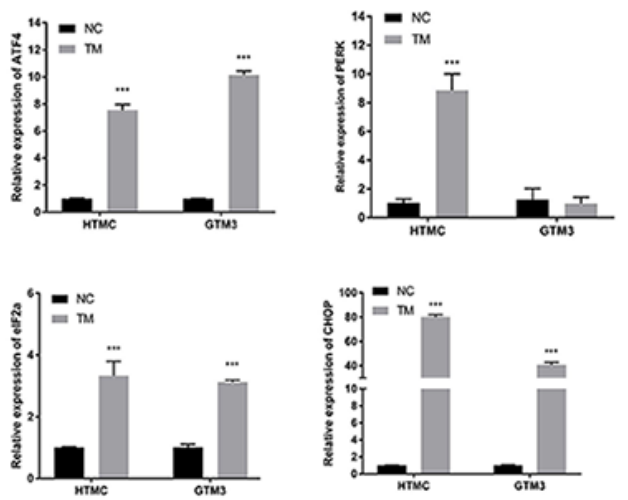

B

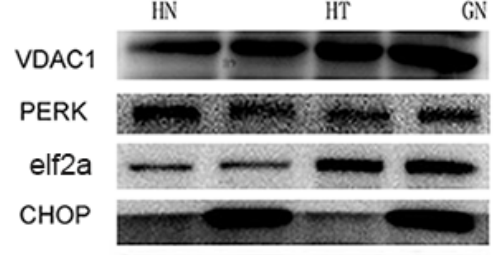

IP3R

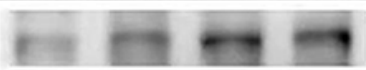

GRP-78

C $\beta$-actin
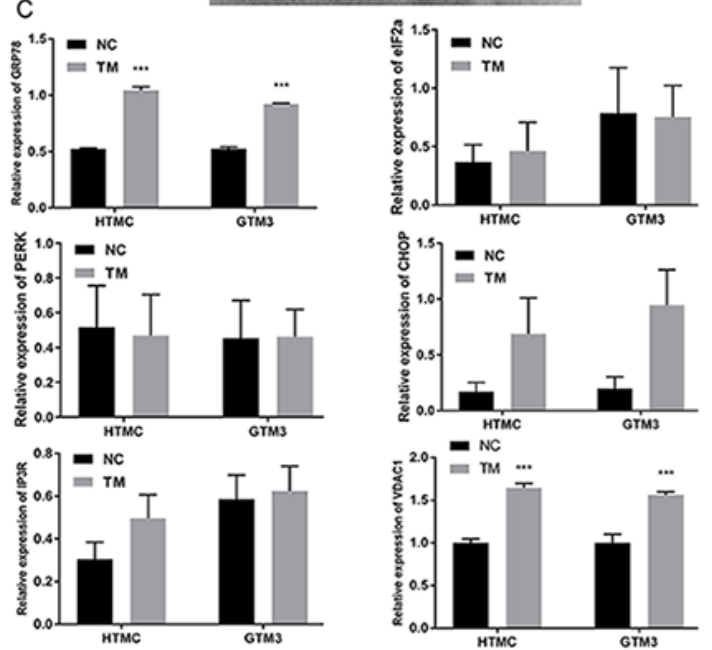

Figure 3. Tunicamycin enhanced GRP78 and activated the PERKelF2A-ATF4 /CHOP pathway.

(A) The mRNA levels of ATF4, PERK, elF2a and CHOP were detected by $\mathrm{qPCR}$ after addition of tunicamycin to HTMC and GTM3 cells. (B) The expression levels of VDAC1, PERK, elF2a, CHOP, IP3R and GRP78 were detected by Western blot after addition of tunicamycin to HTMC and GTM3 cells. (C) Gray value statistics of WB results. ${ }^{* *} p<0.01 ;{ }^{* * *} p<0.001$. NC, negative control (PBS treated group); TM, tunicamycin treated group.

creased in both, HTMC $(p<0.01)$ and GTM3 $(p<0.001)$ (Fig. 1A-1B). This showed that tunicamycin leads to the trabecular meshwork cells' apoptosis.

At the same time, ROS content in trabecular meshwork cells treated with tunicamycin was estimated, and the results showed that $1 \mu \mathrm{M}$ tunicamycin treatment for $24 \mathrm{~h}$ could increase the content of ROS in HTMC cells $(p<0.001)$ and GTM3 cells $(p<0.01)$ (Fig. 2A-2B). These 
A

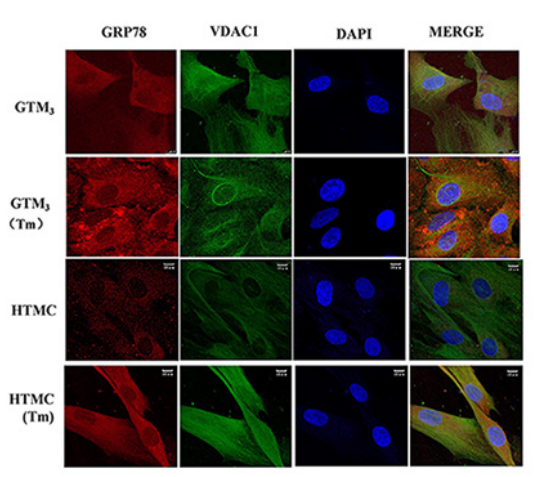

B

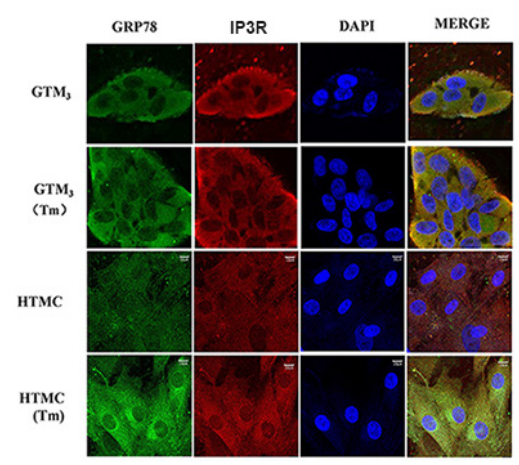

Figure 4. ER protein expression was detected by immunofluorescence.

(A) Expression of GRP78 and VDAC1 and co-localization in HTMC and GTM3 cells after tunicamycin treatment were detected by immunofluorescence after addition of tunicamycin to HTMC and GTM3 cells. (B) Expression of GRP78 and IP3 and co-localization in HTMC and GTM3 cells after tunicamycin treatment were detected results indicated that tunicamycin could induce oxidative stress and apoptosis.

\section{Tunicamycin increased GRP78 and activated the PERK- elF2A-ATF4 /CHOP pathway}

In order to clarify the regulatory mechanism of tunicamycin, we detected expression of GRP78, VDAC1, ATF4, PERK, eIF2a, and CHOP. The qPCR results showed that tunicamycin could significantly increase the mRNA expression of ATF4 $(p<0.001)$, PERK $(p<0.001)$, eIF2a $(p<0.001)$, and CHOP $(p<0.001)$ in HTMC cells (Fig. 3A). And tunicamycin could increase the mRNA expressions of ATF4 $(p<0.001)$, eIF2a $(p<0.001)$ and CHOP $(p<0.001)$ in GTM3 cells. Western blot results showed that expression of GRP-78 $(p<0.001)$ and VDAC1 $(p<0.001)$ in HTMC cells were significantly increased in the tunicamycin treatment group. Expression of GRP-78 $(p<0.001)$ and VDAC1 $(p<0.001)$ in GTM3 cells was also significantly increased in the tunicamycin treatment group (Fig. 3B-3C). Thus, tunicamycin can increase GRP78 expression, as well as activate the PERKeIF2a-ATF4/CHOP pathway.

\section{Regulation of ER protein by tunicamycin}

In order to clarify the regulatory effect of tunicamycin on ER proteins, expressions of GRP78, VDAC1 and IP3 in HTMC and GTM3 cells treated with tunicamycin were detected by immunofluorescence. The results showed that in the absence of external stimuli, GRP78 expression was not high in GTM3 and HTMC, while VDAC1 and IP3 were slightly expressed. After tunicamycin treatment, VDAC1 and IP3 increased with the increase of GRP78 expression level (Fig. 4A-4B). The results showed that ER stress induced by tunicamycin was related to GRP78, VDAC1 and IP3.
A

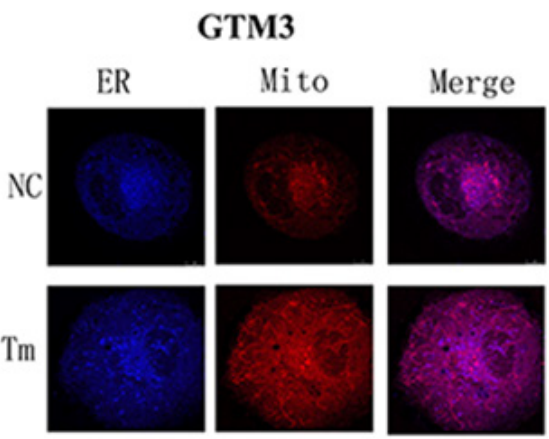

B

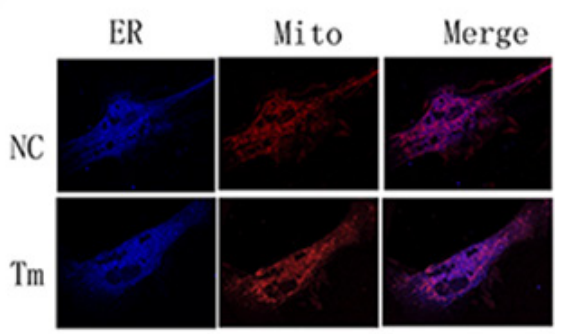

C

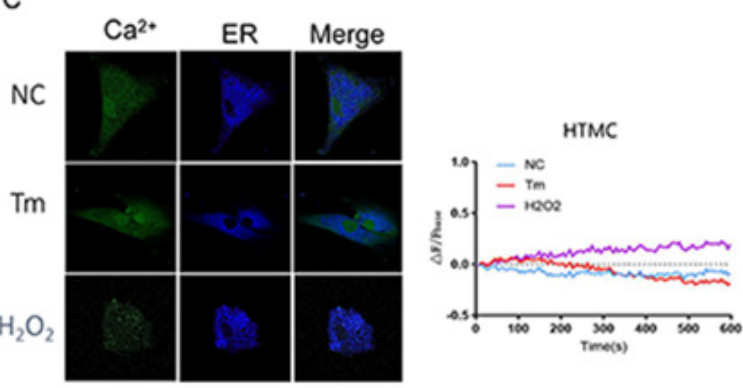

D

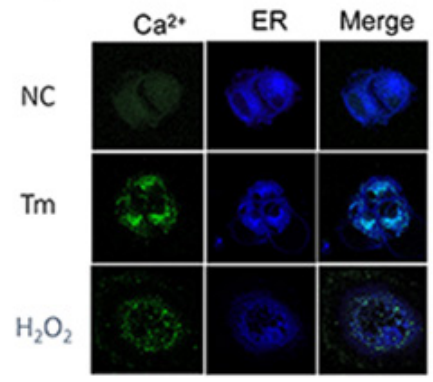

Figure 5. Immunofluorescence detection of mitochondria and ER calcium channels.

(A-B) mitochondrial and ER immunofluorescence results in HTMC and GTM3 cells. (C-D) After treatment of HTMC and GTM3 cells with $\mathrm{PBS}, \mathrm{TM}$ and $\mathrm{H}_{2} \mathrm{O}_{2}$, respectively, the calcium channel and calcium ion current were detected. NC, negative control (PBS treated group); $\mathrm{TM}$, tunicamycin treated group. 
A

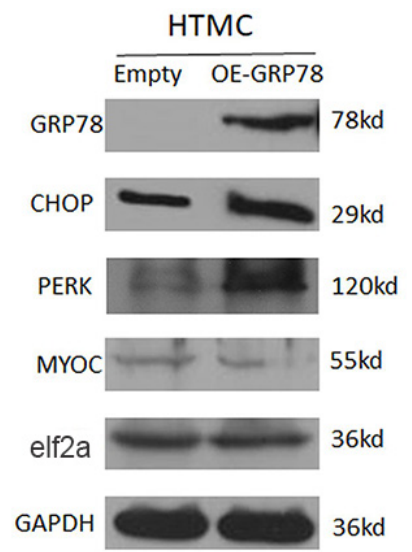

B

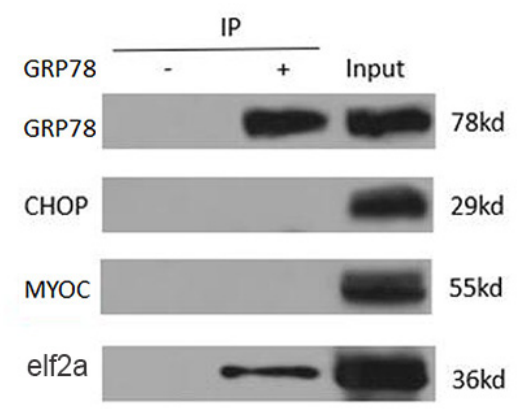

Figure 6. GRP78 protects cells during stress.

(A) After overexpression of GRP78, protein expressions of CHOP and PERK were significantly increased. (B) Co-IP detection revealed that GRP78 protein could bind and interact with the elF2A protein. (C) Apoptosis rate was detected after over-expression of GRP78. Input was total protein, which served as a positive control.

Next, the mitochondria and ER calcium channels of trabecular reticulum cells were examined. The results showed that tunicamycin could cause mitochondrial accumulation (Fig. 5A-5B) and ER enlargement (Fig. 5C5D). The results suggested that tunicamycin could induce ER stress and apoptosis by increasing GRP78 and activating PERK-eIF2A-ATF4 /CHOP.

\section{GRP78 protected cells from ER stress}

In order to further clarify the effect of GRP78 on trabecular meshwork cells, protein expression of CHOP and PERK were increased after overexpressing GRP78. Meanwhile, the Co-IP detection showed that GRP78 could bind and interact with eIF2a (Fig. 6A-6B), suggesting that GRP78 may play a regulatory role by directly binding to eIF2a. Also, the apoptosis rate was decreased after over-expressing GRP78 (Fig. 6C).

\section{DISCUSSION}

The purpose of this study was to clarify the regulatory effect of tunicamycin on ER stress in trabecular reticulum cells and its molecular regulatory mechanism. The results showed that tunicamycin could significantly increase the ROS content and the apoptosis rate of HTMC and GTM3. Tunicamycin could also increase the $\mathrm{Ca}^{2+}$ flow in the cells. However, the protein expressions of CHOP and PERK were significantly increased after overexpression of GRP78, and the co-IP test showed that GRP78 directly bound to eIF2. These results suggest that GRP78 may play a regulatory role by regulating eIF2.

The ER stress response is when the stability of the ER is unbalanced and the physiological function is disturbed. The stable intracellular environment keeps the cell alive and activates the self-protection response mechanism (Kaira et al., 2016; Kaira et al., 2016). When the ER stress response is too strong and too long, the apoptotic pathway can be initiated, which leads to abnormal cell function and finally leads to cell death. When the ER stress response capacity is low, the effective cell protection mechanism is reduced, and the sensitivity of cells to injurious stimuli is increased, which leads to or accelerates cell death (Liu et al., 2020). In our study, it was found that tunicamycin could induce ER stress in trabecular meshwork cells and increased the cell apoptosis rate.

The change in GRP78 expression is considered to be a marker of the ER stress due to unfolded protein response. Transcriptional activity of the GRP78 gene is significantly increased during ER stress response, thus maintaining ER calcium homeostasis, internal environment stability, and cellular protection. GRP78 also reduced sensitivity of cells to killer T cells and prevented cell apoptosis (Peters et al., 2015). Therefore, it has been suggested in recent years that the response of high expression of GRP78 when cells are stimulated may be an important defense mechanism which has a protective effect on cells, thereby prolonging cell survival under various adverse factors. GRP78 also has a protective effect on tumor cells. GRP78 can reduce the lethality of cytotoxic $\mathrm{T}$ cells to tumor cells, promote tumor formation and the development of drug resistance, and prevent tumor cell apoptosis ( $\mathrm{Lu}$ et al., 2020; Borok et al., 2020). Similarly, in our research, expression of GRP78 was increased after treatment with tunicamycin.

Tunicamycin is a kind of protein glycosylation inhibitor, and also is an inducing factor of ER stress in cells. Previous research showed that after garment drug treatment the GRP78 expression quantity increased significantly in NTM (normal trabecular meshwork cells) and GTM (trabecular meshwork cells in patients with glaucoma) cells, , but the increase in GRP78 expression level in GTM cells is still lower than that in NTM, while the NTM and GTM cells under the stimulus of ER stress can produce the corresponding stress reaction (Tsai et al., 2018). However, GTM cells to the ER stress response ability than NTM cells. After treatment with STS, GRP78 protein expression in both, the NTM and GTM cells was down-regulated, and the down-regulation rate in GTM cells was significantly higher than in NTM cells, suggesting that the protective GRP78 protein was down-regulated in GTM cells, the intracellular pro-survival factors were decreased, and the ER defense function was damaged, leading to increased sensitivity of GTM cells to apoptosis stimulation (Chern et al., 2019; Shah et al., 2019). In summary, the results of this study showed that expression of GRP78 in GTM cells decreased and the response to ER stress stimulation was low, indicating that the ER stress response defense system was damaged, which increased the sensitivity of cells to traumatic stimulation, and may accelerate or lead to trabecular cell death. In this study, tunicamycin was added to HTMC and GTM3 cells cultured in vitro, and it was found that the expression of 
GRP78, VDAC1, ATF4, PERK, eIF2a, and CHOP were increased after addition of tunicamycin.

\section{CONCLUSION}

This study demonstrated that tunicamycin can induce oxidative stress in trabecular meshwork cells, that increased GRP78 expression can protect cells during the stress process, and that GRP78 may play a protective role by regulating eIF2.

\section{Data Archiving}

Not applicable.

\section{Acknowledgements}

None.

\section{Statement of Ethics}

Not applicable.

\section{Authorship}

CP was dedicated to the study concepts; HY carried out the study design and manuscript review; JL managed the definition of intellectual content and manuscript editing; FC undertook the literature research, experimental studies, statistical analysis, data analysis and manuscript preparation; XZ performed the clinical studies and data acquisition. All authors have read and approved this article.

\section{REFERENCES}

Ashworth BE, Toh TY, Eri R, Hewitt AW, Cook A (2018) Uteroglobin and FLRG concentrations in aqueous humor are associated with age in primary open angle glaucoma patients. BMC Ophthalmol 18: 57. https://rdcu.be/cHyFF

Bailly C, Waring MJ (2019) Pharmacological effectors of GRP78 chaperone in cancers. Biochem Pharmacol 163: 269-278. https://doi. org/10.1016/j.bcp.2019.02.038

Borok Z, Horie M, Flodby P, Wang H, Liu Y, Ganesh S, Firth AL, Minoo P, Li C, Beers MF, Lee AS, Zhou B (2020) Grp78 Loss in epithelial progenitors reveals an age-linked role for endoplasmic reticulum stress in pulmonary fibrosis. Am J Respir Crit Care Med 201: 198-211. https://doi.org/10.1164/rccm.201902-0451OC

Chern YJ, Wong JCT, Cheng GSW, Yu A, Yin Y, Schaeffer DF, Kennecke HF, Morin G, Tai IT (2019) The interaction between SPARC and GRP78 interferes with ER stress signaling and potentiates apoptosis via PERK/eIF2alpha and IRE1alpha/XBP-1 in colorectal cancer. Cell Death Dis 10: 504. https://rdcu.be/cHyF5

Ibrahim IM, Abdelmalek DH, Elfiky AA (2019) GRP78: A cell's response to stress. Life Sci 226: 156-163. https://doi.org/10.1016/j. lfs.2019.04.022

Kaira K, Toyoda M, Shimizu A, Mori K, Shino M, Sakakura K, Takayasu Y, Takahashi K, Oyama T, Asao T, Chikamatsu K (2016) Expression of ER stress markers (GRP78/BiP and PERK) in patients with tongue cancer. Neoplasma 63: 588-594. https://doi. org/10.4149/neo 2016_412

Kaira K, Toyoda M, Shimizu A, Shino M, Sakakura K, Takayasu Y, Takahashi K, Asao T, Chikamatsu K (2016) Expression of ER stress markers (GRP78/BiP and PERK) in adenoid cystic carcinoma. Acta Otolaryngol 136: 1-7. https://doi.org/10.3109/00016489 .2015 .1083120

Lee, AS (2005) The ER chaperone and signaling regulator GRP78/BiP as a monitor of endoplasmic reticulum stress. Methods 35: 373-381. https://doi.org/10.1016/j.ymeth.2004.10.010

Lu G, Luo H, Zhu X (2020) Targeting the GRP78 pathway for cancer therapy. Front Med (Lausanne) 7: 351. https://doi.org/10.3389/ fmed.2020.00351

Liu K, Tsung K and Attenello FJ (2020) Characterizing cell stress and GRP78 in glioma to enhance tumor treatment. Front Oncol 10: 608911. https://doi.org/10.3389/fonc.2020.608911

Peters JC, Bhattacharya S, Clark AF, Zode GS (2015) Increased endoplasmic reticulum stress in human glaucomatous trabecular meshwork cells and tissues. Invest Ophthalmol Vis Sci 56: 3860-3868. https://doi.org/10.1167/iovs.14-16220

Shah A, Chao JF, Cristina LQ, Chang RCC (2019) Oxyresveratrol exerts ATF4- and Grp78-mediated neuroprotection against endoplasmic reticulum stress in experimental Parkinson's disease. Nutr Neurosci 1-16. https://doi.org/10.1080/1028415X.2019.1613764

Shen J, Chen X, Hendershot L, Prywes R (2002) ER stress regulation of ATF6 localization by dissociation of BiP/GRP78 binding and unmasking of Golgi localization signals. Dev Cell 3: 99-111. https:// doi.org/10.1016/S1534-5807(02)00203-4

Sun B, Yang S, Li S, Hang C (2018) Melatonin upregulates nuclear factor erythroid-2 related factor $2(\mathrm{Nrf} 2)$ and mediates mitophagy to protect against early brain injury after subarachnoid hemorrhage. Med Sci Monit 24: 6422-6430. https://doi.org/10.12659/ MSM.909221

Tsai YL, Ha DP, Zhao H, Carlos AJ, Wei S, Pun TK, Wu K, Zandi E, Kelly K, Lee AS (2018) Endoplasmic reticulum stress activates SRC, relocating chaperones to the cell surface where GRP78/CD109 blocks TGF-beta signaling. Proc Natl Acad Sci USA 115: E4245E4254. https://doi.org/10.1073/pnas.1714866115

Xu YP, Sui XL, Zhang AS, Ye L, Gu FJ, Chen JH (2017) Monocytes, endoplasmic reticulum stress and metabolomics in dogs with multiple organ dysfunction syndrome treated by continuous venovenous hemodiafiltration. Oncotarget 8: 34992-5008. https://doi. org/10.18632/oncotarget.16533

Zhu W, Godwin CR, Cheng L, Scheetz TE, Kuehn MH (2020) Transplantation of iPSC-TM stimulates division of trabecular meshwork cells in human eyes. Sci Rep 10: 2905. https://doi.org/10.1038/ s41598-020-59941-0 